Culture et histoire dans l'espace roman

$6 \mid 2011$

Figures du pouvoir dans la littérature hispanoaméricaine

\title{
Lorsque l'écriture affronte les fantômes de la dictature
}

Le pouvoir des mots dans la lutte contre la disparition dans Purgatorio de Tomás Eloy Martínez

\section{Lucie Valverde}

\section{(c) OpenEdition}

\section{Journals}

Édition électronique

URL : https://journals.openedition.org/cher/9290

DOI : $10.4000 /$ cher.9290

ISSN : 2803-5992

Éditeur

Presses universitaires de Strasbourg

Édition imprimée

Date de publication : 30 juin 2011

Pagination : 281-290

ISBN : 978-2-35410-033-9

ISSN : 1968-035X

\section{Référence électronique}

Lucie Valverde, "Lorsque l'écriture affronte les fantômes de la dictature », reCHERches [En ligne], 6 |

2011, mis en ligne le 17 décembre 2021, consulté le 27 janvier 2022. URL : http://

journals.openedition.org/cher/9290 ; DOI : https://doi.org/10.4000/cher.9290

\section{cc) (1) (ㅇ)}

Ce(tte) œuvre est mise à disposition selon les termes de la Licence Creative Commons Attribution -

Pas d'Utilisation Commerciale - Partage dans les Mêmes Conditions 4.0 International. 


\title{
Lorsque l'écriture affronte les fantômes de la dictature: le pouvoir des mots dans la lutte contre la disparition dans Purgatorio de Tomás Eloy Martínez
}

\author{
LUCIE VALVERDE
}

Université d'Angers

\begin{abstract}
C'est en tant que journaliste que Tomás Eloy Martínez a commencé à côtoyer la sphère du pouvoir politique, et a aussitôt entrevu le pouvoir que pouvaient détenir les mots. Ces premières expériences de lutte de pouvoir l'amèneront à se lancer dans son premier "duel de versions narratives", en publiant La novela de Perón en 1985. C'est une autre impulsion très personnelle qui l'amènera à l'écriture de Purgatorio, qui revisite le temps de la dictature militaire argentine de 1976, dans l'espoir d'exorciser les années d'exil qu'il a connues, à l'instar de bon nombre de ses compatriotes. Ces années volées, irrécupérables, Martínez va tenter de les reconstruire à travers la fiction, en faisant réapparaître ce qui a disparu, en redonnant vie à travers la littérature à toutes les possibilités que l'Histoire a anéanties.

Purgatorio constitue une lutte menée par un contre-pouvoir artistique vital contre la capacité de destruction d'un pouvoir dictatorial mortifère. Dans ce combat, les mots sont des armes qu'utilisent aussi bien les autorités civile et militaire dans leur construction d'une irréalité sinistre (propagande, censure, illusions diverses), que les contre-pouvoirs présents dans le roman, entre autres les arts, représentés par le maître de l'illusion Orson Welles et par le narrateur (double de l'auteur) dans sa volonté de corriger la réalité à travers la fiction. Ces contre-pouvoirs vont ainsi corriger la réalité créée par les mots de la dictature et défaire son œuvre de mort. Cette déroute de l'autoritarisme se ressent notamment à travers la trajectoire vitale de la protagoniste, Emilia,
\end{abstract}

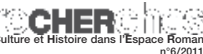


dont le mari, Simón, fait partie des disparus (et réapparaît mystérieusement 30 ans plus tard). En effet, l'évolution d'Emilia constitue non seulement une rébellion contre le joug paternel et contre le pouvoir de la dictature, mais aussi un formidable message de vie et d'espoir.

Afin d'analyser les rapports de pouvoir présents dans Purgatorio, nous nous appuierons ici sur la réflexion menée par Marie-Claire Kerbrat dans Figures du pouvoir, qui étudie notamment les mots permettant de se figurer le pouvoir, en l'occurrence les deux noms du maître: magister et dominus. Une distinction fondamentale sépare ces deux «maitres» :

Le maitre-magister s'oppose diamétralement au dominus: son but est d'éduquer l'élève, c'est-à-dire, étymologiquement (educere, exducere: faire sortir, conduire hors de), de le libérer. [...] Tandis que l'esclave est prisonnier, non seulement de son maitre, mais de la servitude qui l'instrumentalise irrémédiablement, l'élève s'affranchit progressivement jusqu’à devenir l'égal de son maître (Kerbrat 1994:7).

Nous verrons donc comment s'affrontent dans Purgatorio ces deux types de maîtres autour d'une lutte pour le contrôle du discours.

\section{Un pouvoir tyrannique: le docteur Dupuy}

Bien qu'ayant des objectifs fondamentalement opposés, le pouvoir de la Junte et le contre-pouvoir artistique sont liés par leurs connaissances respectives en termes de fiction et de vérité. La différence essentielle qui les sépare réside dans l'attitude que chacun observe vis-à-vis du rapport entre discours et vérité: si ce rapport est remis en question par le roman, c'est le discours qui est fondateur de vérité pour les régimes autoritaires. C'est le personnage du docteur Dupuy, le père d'Emilia, qui assume ici ce rôle de créateur de la réalité conforme aux intérêts du régime: "Los comandantes delegaban en Dupuy el cuidado de la irrealidad y se ocupaban sólo de la represión armada» (Martínez 2008: 268).

Pure création littéraire, Dupuy est un personnage surnuméraire: il n'a jamais existé, et n'a pas de modèle dans le monde de référence. Il incarne cependant le mode de pensée de la dictature, puisqu'il en est à la fois l'idéologue et le porte-voix. C'est à travers une presse muselée ou complice qu'il répand sa propagande:

En aquellos finales de 1978 los diarios y las radios publicaban lo que se les permitía. (...) a esas alturas la sumisión y el miedo se habían tornado 
costumbre. (...) Total, los lectores fingían ignorancia y repetían que el silencio es salud (268).

Mais Dupuy fait plus que veiller à la diffusion de la propagande du régime: non content de contrôler l'opinion, il possède en outre sa propre revue, La República, dans laquelle il «fait» l'Histoire: «La revista no sólo anunciaba con anticipación los golpes militares; era también el viento que los impulsaba» (32-33).

Omniprésent, son pouvoir s'exerce sur tous les plans de la vie publique: personnage odieux s'il en est, il incarne le dominus par excellence. Son champ d'action est illimité: il est indispensable et donc intouchable pendant la dictature, sa position d'éminence grise faisant de lui l'un des piliers du régime.

Opportuniste, il fait passer avant tout ses intérêts personnels: ce personnage est caractérisé par l'omniprésence de son soi, sans extériorité, sans Autre, il impose ses décisions. Calculateur et machiavélique, il représente la corruption qui gangrène le pouvoir ainsi que son hypocrisie; il ne considère les êtres qui l'entourent que comme des adjuvants ou des opposants, des pions sur un échiquier qu'il déplace selon ses besoins. Lorsque frappe l'adversité, il lui faut nécessairement un coupable, quitte à l'inventer. Il orchestre donc la «fausse guerre» menée par la dictature contre ces ennemis imaginaires, boucs émissaires tout désignés:

Emilia nunca sabía de cuáles enemigos hablaba el padre, porque un día eran los Montoneros y el Ejército Revolucionario del Pueblo y al otro día, como ya los habían exterminado, eran las brigadas de un almirante que conspiraba contra la Anguila, o una emisaria del gobierno norteamericano, o Pinochet que amenazaba invadir las islas del canal Beagle, o los intermediarios corruptos que paralizaban las usinas nucleares. Cuando se replegaban unos, avanzaban los otros, y a veces ninguno se replegaba (154-155).

Dupuy incarne aussi le mal qui contamine: son discours imprègne et détruit. Ainsi, son action de propagande induit une perte de mémoire et de repères symptomatique de l'Argentine de l'époque. Mal inhérent à la dictature, cette amnésie est métaphorisée par Ethel, la femme de Dupuy. Cet état de fait ne semble pas déplaire à ce dernier, puisqu'il espère aussi "[domar] para siempre» (181) sa fille Emilia, l'animalisant donc dans un premier temps, et poussant la déshumanisation à son extrême en la réifiant, comme il a déjà réifié sa femme: «la convertirían en una planta como la madre» (181). Il l'amène donc à un état de conscience toujours moindre, comme il réduit la société à un espace vide de pensée. Ainsi, Dupuy est un 
dominus absolu: il obtient l'obéissance du pays, mais aussi de ses proches, dominés par la crainte que leur inspire «su lengua envenenada» (181).

Le discours est donc bel et bien l'arme de Dupuy; entre maitrise du discours (par l'omission et la falsification, la propagande et l'historiographie) et intimidation physique, il satisfait la haine, la cupidité et la vanité, en parfait dominus.

C'est le portrait d'un homme terrifiant qu'on nous dresse donc ici: incarnation de l'autoritarisme d'extrême droite, il concentre sur lui seul toute la noirceur du régime. Même la Anguila (Videla) fait bien pâle figure à ses côtés. Nul doute qu'Emilia ait été réduite en miettes par un père aussi tyrannique; toutefois, si Dupuy semble être dans Purgatorio le créateur de la réalité funeste de la dictature, il va se heurter à plus grand démiurge que lui: l'auteur, Tomás Eloy Martínez, qui d'un coup de plume va rayer ce personnage de la carte et retourner ses propres armes contre lui. S'engage en effet entre la fiction et la réalité une lutte féroce dans laquelle Dupuy (et à travers lui, «l'œuvre» de la dictature) va affronter un ennemi de taille: le contre-pouvoir des arts et de l'imagination, incarné entre autres par Orson Welles.

\section{Un contre-pouvoir artistique}

La raison d'être de ce roman est exprimée à plusieurs reprises par le narrateur:

Esos treinta años de separación [...] repiten de algún modo el vacío de los treinta años que pasé fuera de mi país y al que esperé encontrar, cuando volviera, tal como lo había dejado. Sé que se trata de una ilusión, ingenua como todas las ilusiones, y tal vez fue eso lo que me atrajo, porque los años perdidos nunca dejaron de atormentarme y si los cuento, si imagino la vida de cada día que no viví, quizá - me dije - pueda exorcizarlos. Quería recordar lo que no vi, contar la vida que hubiera tenido cada día [...] (240).

Tel est le projet qui sous-tend le roman : recréer une réalité dérobée par la dictature. Martínez et Dupuy vont donc s'affronter et poursuivre un même dessein, bien qu'avec une finalité diamétralement opposée; en effet, Dupuy résume ainsi son ambition: «Si ustedes fracasaron con la realidad, yo no tengo por qué fracasar con la ilusión» (217).

En d'autres termes, prononcés cette fois-ci par le narrateur, double de l'auteur: "Voy a devolver los hechos a la realidad de la que salieron" (289). Cette phrase est à double sens: au sens premier, ramener Emilia à la «raison», puisqu'elle nie encore l'évidence de la mort de son mari; et dans 
un second, il s'agit des intentions du narrateur, puisque le roman s'achève sur son projet d'écrire le roman que nous venons de terminer, un «romanréparation» qui retrace une ligne de vie, celle dont la trajectoire a été brisée par le coup d'État.

Ainsi, si Dupuy avait créé une illusion mortifère, celle créée par Martínez et soutenue par les porte-parole de l'art dans Purgatorio est une illusion pleinement vitale, le pouvoir formidable de l'imagination va défaire l'œuvre meurtrière de la dictature, et libérer Emilia (et donc l'Argentine, dont elle est la métaphore) de ces funestes années: «si nos entregáramos a la busca de lo que no existió y lo encontráramos, entonces habríamos vencido a la muerte» (241).

Vaincre la mort, c'est vaincre la dictature et son œuvre; en effet, elle n'a semé que la mort et l'exil (l'exil étant, comme le rappelle l'auteur, un châtiment aussi sévère que la mort pour les Grecs). Ce projet sera mené à bien en utilisant certaines armes du pouvoir autoritaire, c'est-à-dire la maîtrise du discours et de l'illusion, mais cette fois-ci le discours ne sera ni destruction, ni enfermement, mais création et libération: écrire est déjà en soi un acte de liberté, et la finalité de l'écriture est ici de libérer. En effet, le contre-pouvoir artistique détient la clé qui permet de passer d'une réalité à l'autre: ce sésame, c'est le pouvoir des mots à travers lesquels on convoque une autre réalité.

Ainsi, si le pouvoir autoritaire présente toutes les caractéristiques du dominus, le contre-pouvoir artistique est aussi un maître, mais un maître magister, qui libère au lieu d'asservir.

Dans Purgatorio vont donc s'affronter le dominus Dupuy, représentant de tous les domini de l'Argentine de l'époque, et un certain nombre de magistri. Plusieurs démiurges vont ainsi croiser le fer avec la réalité créée par la dictature, l'un d'eux sous les traits d'Orson Welles lui-même. Dupuy et Welles vont s'affronter dans une joute verbale menée, dominée de bout en bout par le cinéaste, subie par un Dupuy médusé, véritable pantin entre les mains de Welles: le manipulateur manipulé essuie alors une cuisante défaite.

Au seuil de cette scène, on nous présente un Dupuy au pouvoir amoindri, car on porte atteinte à son statut de dominus en rappelant les limites géographiques de son pouvoir:

lo que usted dice sólo influye, por desgracia, dentro del país. [...] Su columna ha sido traducida y enviada por nuestras embajadas a los diarios del extranjero, dijo el marino. Hemos ofrecido fortunas para que la publiquen. 
La mayoría nos ha contestado que no la quieren, ni aun como espacio de publicidad (201).

Son discours étant refusé tout net à l'étranger, Dupuy cherche à «mondialiser» son emprise, et il choisit pour cela de faire réaliser par un porte-parole au discours reconnu de par le monde un film de propagande autour du mondial de football: Orson Welles. Dupuy évoque alors ce qui les unit: "Yo hablo su mismo lenguaje» (204) Ce même langage, c'est celui de l'imagination, de l'illusion.

Mais Dupuy commettra deux erreurs, découlant de sa méconnaissance d'une part du caractère du cinéaste, selon lui «más un buey aburrido que un toro de lidia» (204), et d'autre part de son œuvre elle-même, dont il n'a qu'une vague idée (on mentionne notamment qu'il ne prend pas le temps de visionner $F$ for fake, film sur la falsification et la magie).

Dupuy sous-estime donc son interlocuteur, et se comporte dès le départ en dominus, comme l'indiquent ses réflexions: "Si Welles obedecía sus órdenes » (204), «Él, Dupuy, iba a elegir la música », " convencería a Welles », «Orson le iba a dar las gracias de rodillas» (205). Mais le rapport de force va s'inverser au cours de l'échange avec le cinéaste; déjà, lorsque Dupuy demande à le rencontrer, Welles s'avère être un adversaire redoutable:

Díganle a ese hombre que venga a verme. Tuve a Capone, a Lucky Luciano y a Costello persiguiéndome para que les hiciera películas. Me los saqué de encima y sigo vivo (207).

Welles ne s'abaisse pas même à prononcer le nom de Dupuy (tout comme Martínez ne s'abaisse pas à prononcer le nom de Videla, surnommé la Anguila). D'emblée, il ne semble pas de ceux sur lesquels on fait pression, puisque même de redoutables mafieux ne l'ont pas fait plier.

Dès le début de leur entretien, il se révèle impossible à dominer; tout d'abord, il refuse de parler dans la langue de son interlocuteur (bien qu'il la maîtrise parfaitement, comme on nous l'a précisé plus haut), ce qui démontre déjà l'indépendance de son discours. De même, à la première tentative de familiarité de Dupuy, Welles se positionne clairement:

¿Puedo llamarte Orson?, dijo Dupuy. Nos conocimos hace diez años en el camarín de Antonio Bienvenida.

Llámame Orsten, dijo Welles, sin dar señales de recordar a Bienvenida. Así me llamaba Lucky Luciano, Orsten. Y yo a él lo llamaba Charlie. ¿Puedo llamarte Charlie? (207). 
Faisant mine de ne pas reconnaître Dupuy, il lui refuse la proximité réclamée, tout en annonçant déjà en filigrane l'issue de cette conversation: en assimilant Dupuy à Lucky Luciano (à un criminel donc), on peut supposer que Welles refusera de réaliser le film que lui demande Dupuy, comme il l'avait refusé à Luciano par le passé. Déstabilisé, Dupuy éprouve alors le besoin de réaffirmer son statut de maître: «Él, Dupuy, era un maestro de la política; Welles, menos que un aprendiz. Y, a la inversa, Dupuy llevaba años sin pisar una sala de cine» (208). Tout en déclarant sa position de maître («maestro»), Dupuy saisit pourtant ce qui le sépare du cinéaste: ils ont beau parler le même langage, ils ne sont pas du même monde. A cet instant, Welles montre à son tour être conscient de ce qui l'unit à ce dominus, à savoir la maîtrise de l'illusion:

Aquí, en estos papeles, he leído que en tu país hay magos, ilusionistas, Charlie. ¿Es verdad? Como sabrás, yo soy más un ilusionista que un director de cine. [...] aquí leo [...] que los magos de tu gobierno hacen desaparecer a la gente en las calles (209).

Dupuy ira alors de surprise en consternation, jusqu'au coup fatal que lui assène Welles:

Te hago un trato, Charlie. Yo pongo mi magia en ese documental, tú me pagas con tu magia. [...]

Te hago la película gratis, con el mejor mundial de fútbol que se haya visto, y tú con tus comandantes hacen aparecer a los desaparecidos (214).

Welles proclame ainsi ce qui le différencie de Dupuy: leurs magies ne sont pas équivalentes, la sienne est créatrice, celle de Dupuy destructrice. Il s'en distingue enfin en se déclarant incorruptible: il refuse l'argent proposé, laissant entendre qu'il ne mettra pas sa magie au service d'une cause contraire à son éthique. Dupuy, en tant que dominus, n'a pas coutume de se voir opposer un refus aussi cinglant, lui qui tyrannise jusqu'à ses plus proches parents, comme sa fille Emilia; celle-ci va pourtant également se rebeller contre le joug paternel et s'émanciper grâce au pouvoir de l'imagination.

\section{Entre dominus et magister : la trajectoire d'Emilia}

Esclave de son père (et par métonymie, du pouvoir dictatorial), Emilia va peu à peu se libérer de son emprise. Nous constaterons en effet que l'émancipation de "la niñita a la que el doctor Dupuy había destrozado" (233) coïncide avec son passage de l'emprise d'un dominus à sa libération par des magistri. 
Dupuy enferme psychologiquement sa victime: Emilia passe «de celda en celda; ésta, la de su padre, era la más amarga» (223). Mais il la cloître aussi littéralement, utilisant notamment pour la torturer la terreur que lui inspirent les miroirs: il l'enferme dans une pièce entièrement tapissée de miroirs, dans laquelle Emilia «Se sintió dentro de una tumba, sellada para siempre» (283). L'asservissement d'Emilia atteint alors son paroxysme, mais aussi son point de rupture. À cette époque, elle est soumise au point d'adopter le discours de son père; ainsi, lorsqu'elle reçoit un tract dénonçant les morts semés par la dictature, voici sa réaction: «Eran infamias. Todas las revistas decían que los exiliados estaban calumniando al país. Este volante era la prueba. Lo partió en dos y lo arrojó al piso» (32).

De même, peu après la disparition de Simón, alors qu'elle est au cinéma, une femme, Ema, l'approche et tente de la convaincre de la mort de Simón. Emilia la rejette et l'insulte, montrant combien elle est imprégnée du discours de son père: "No te creo, dijo Emilia. Sos una subversiva hija de puta» (30). Nous sommes plus tard confortés dans cette idée, puisqu'on nous fait entendre que les idées d'Emilia sur les subversifs lui viennent de son père:

Más de una vez le había oído decir a su padre que los subversivos, ya diezmados, vendían cualquier historia para llevar gente a su causa. La desconocida era uno de ellos y, aunque Emilia descartaba que le mentía, la imagen de Simón yaciendo como un perro se le quedó clavada mucho tiempo (31).

Le doute pointe donc dans l'esprit d'Emilia, mais bien qu'elle ne pense pas qu'Ema lui ait menti, elle exprime pourtant clairement "de quel côté" elle se trouve, s'incluant dans le "nous» des sympathisants au régime, ou tout du moins des passifs face à la dictature: «A los que no estamos metidos en nada no nos van a hacer nada» (31).

Bien qu'Emilia nie alors avec force tout point commun avec Ema, elles ont des expériences similaires, toutes deux étant veuves de disparus. D’autre part, la ressemblance phonique des deux prénoms ne peut être fortuite: Ema semble un raccourci d'Emilia, de même que l'histoire d'Ema semble être un condensé en quelques pages de l'histoire d'Emilia (bien qu'elles connaissent une fin diamétralement opposée, tragique pour Ema, puisqu'elle paiera de sa vie d'avoir voulu approcher Emilia). Mais Emilia refuse d'admettre l'évidence, et reste dans l'ambivalence face à son père.

Toutefois, elle réagit enfin après qu'il l'enferme dans la salle aux miroirs; elle s'éloigne alors physiquement de lui, abandonnant la maison familiale et retournant à son appartement de jeune mariée. Ainsi, elle fait un premier 
pas vers sa libération, elle "choisit» Simón en retournant là où ils vécurent heureux ensemble, se dégage de l'emprise de son dominus.

Elle se rebelle ensuite clairement en s'unissant aux grands-mères de la place de mai; mais c'est alors qu'elle s'exile à la recherche de Simón, et que commence "su purgatorio amoroso" (285), favorisé par l'intervention déguisée de son père, dans une stratégie pour éloigner de lui sa fille, devenue gênante. Ainsi, l'éloignement physique d'Emilia ne constitue pas une libération, puisqu'elle souffre du vide que constituent l'exil et l'absence de son mari (ce vide étant caractéristique du purgatoire): elle subit encore «la sombra ominosa del padre» (104).

C'est précisément la disparition de cette ombre qui va précipiter la libération d'Emilia. Le narrateur-auteur se fait alors magister (n'oublions pas qu'Emilia, personnage de papier, est soumise aux volontés de son auteur), et à travers les mots va la rendre heureuse, dans ce qu'il appelle lui-même «un acto de justicia literaria » (240). En effet, une fois le dominus mort (une mort décidée par l'auteur, Dupuy étant un personnage surnuméraire), la présence de Simón se fait si impérieuse qu'Emilia la ressent dans chaque fibre de son être, et c'est ainsi qu'elle va le retrouver. L'auteur conduit ainsi son personnage hors du purgatoire: sa liberté en tant qu'écrivain le lui permet. Mais c'est Simón, deuxième magister, qui achèvera ce travail de libération; en amenant Emilia à faire le bilan de sa vie, il lui fait prendre conscience de la permanence de son statut passif de victime:

La vida que estás viviendo te rebaja. Vi la montaña de cupones inútiles para comprar lo que nunca vas a consumir [...]. Y las tarjetas para el bingo. Y las uñas esculpidas. Y las amigas que elegís. En vez de ser tu espejo son tu humillación. ¿Qué has hecho con tu vida, Emilia?

Nada, eso es lo malo. No he hecho nada. Es mi vida la que lo ha hecho todo conmigo (166-167).

La fin du roman constitue une ouverture totale: si auparavant, Emilia a vécu dans une tombe lorsqu'elle était sous la coupe de son père, et a même été la tombe de Simón pendant son long purgatoire, la fin de Purgatorio (dans les deux sens du terme: fin du roman, mais aussi du purgatoire personnel d'Emilia) constitue une explosion de vie: Emilia s'est dégagée complètement de l'emprise de Dupuy, Simón veut sortir de sa tombe et libère le corps d'Emilia, à nouveau disponible pour toutes sortes de plaisirs, y compris charnels. Emilia est heureuse et comblée, elle a atteint son paradis. 


\section{Conclusion}

C'est donc une fin pleine d'espoir, une libération matérialisée par le voyage que décident de faire Simón et Emilia; dorénavant rien ne pourra s'opposer à eux, pas même l'étroitesse du fleuve sur lequel ils ont décidé de naviguer : "Se va a volver ancho para nosotros» (291).

Ainsi, le roman rend toute chose possible, mais avec une tonalité positive cette fois-ci, contrairement au début du roman; il s'oppose notamment $\mathrm{au}$ «En aquellos tiempos todo era posible» (116) relatif à la cruauté invraisemblable du régime autoritaire. Si le pouvoir de la dictature et de ses domini a donné ses chances à l'impossible en permettant d'atteindre des sommets de violence et d'horreur, le pouvoir des mots des magistri donne également ses chances à l'impossible et met ainsi en déroute ce pouvoir mortifère en défaisant son œuvre. Grâce au pouvoir de l'imagination, Martínez rend vie à tout ce qui aurait pu être mais n'a pas été, et va ainsi combler ce vide et conjurer la douleur de l'absence. Ou comme le résume Carlos Fuentes: «porque la historia ha sido lo que es, la literatura nos ofrece lo que la historia no siempre ha sido».

\section{Bibliographie}

Fuentes, Carlos, 2009, «Los desaparecidos de Tomás Eloy», El País, 21.03.2009.

Jouve, Vincent, 2004, L'effet-personnage dans le roman, Paris, Presses Universitaires de France, coll. "écriture".

Kerbrat Marie-Claire, Le Gall Danielle, Leliepvre-Botton Sylvie, 1994, Figures du pouvoir, Paris, Presses Universitaires de France, coll. "Major".

Martínez, Tomás Eloy, 1985, La novela de Perón, Buenos Aires, Legasa.

Martínez, Tomás Eloy, 2008, Purgatorio, Buenos Aires, Alfaguara. 